\title{
MULTIPLICATIVELY INVARIANT SUBSPACES OF BESOV SPACES
}

\author{
BY
}

PER NILSSON

\begin{abstract}
We study subspaces of Besov spaces $\dot{B}_{p}^{s, q}$ which are invariant under pointwise multiplication by characters. The case $s>0$ is completely described, and for the case $s<0$ we extend known results.
\end{abstract}

Multiplicatively invariant subspaces of Besov spaces. The study of subspaces of the homogeneous Besov spaces $\dot{B}_{p}^{s, q}$ invariant for multiplication by characters was initiated by R. Johnson [4], [5]. In this paper we will simplify and improve some of his results and we will also settle some points left open in [4]. More generally if $X$ is any Banach space of (tempered) distributions we associate with $X$ several multiplicative invariant subspaces $\pi \cdot X, \pi_{c} X, \pi_{\omega(h)} X, \pi_{s} X=\pi_{\left(1+|h|^{2}\right)^{3 / 2}} X$. In the case of Besov spaces with $s>0$ we find that $\pi_{s} \dot{B}_{p}^{s, q}=\pi \cdot \dot{B}_{p}^{s, q}$ (which is Theorem 1.1 in [4]) while $\pi_{s-\varepsilon} \dot{B}_{p}^{s, q}=0$ if $\varepsilon>0$. It turns out that the cases $s=0$ and $s<0$ behave quite differently. For instance if $s<0$ we prove $\pi_{c} \dot{B}_{p}^{s, q}=0$ if $s<-n / p^{\prime}$ or $s=n / p^{\prime}, q=\infty$. In [4] this is done only for the case $1<p<2$. We also remark that these techniques have applications to Fourier multipliers between $L^{p}$-spaces. See [4], [5].

While in [4] the Besov spaces are defined using finite differences we will use the definition of Besov spaces depending on a general partition on the Fourier side as in [8]. From this definition it is apparent that the origin on the Fourier side plays a particular role. Since multiplication by a character means translation after Fourier transform this puts a heavy restriction on the multipliers.

I wish to express my gratitude to Professor J. Peetre for his guidance during my work on this paper.

0. Conventions. All function or distribution spaces are considered on $\mathbf{R}^{n}$. Likewise all integrals without integration limits are taken over all $\mathbf{R}^{n}$. In particular, $L^{p}$, where $1 \leqslant p \leqslant \infty$, denotes the Lebesgue space of measurable functions $f$ such that the norm $\|f\|_{p}=\left(\int|f|^{p} d x\right)^{1 / p}$ is finite. We let $\Re$ be the space of bounded measures on $\mathbf{R}^{n}$ and denote its norm likewise by $\|\cdot\|_{1}$. As usual $\mathcal{S}$ is the space of rapidly decreasing functions and its dual, the space of tempered distributions, will be denoted by $\mathcal{S}^{\prime}$. Similarly $\mathscr{D}^{\prime}$ is the space of all (L. Schwartz) distributions. The relation $X \subset Y$, where $X$ and $Y$ are topological vector spaces, means that we have

Received by the editors March 31, 1980.

1980 Mathematics Subject Classification. Primary 46E35; Secondary 46F05.

Key words and phrases. Besov spaces, Morrey spaces, pointwise multipliers.

(C) 1981 American Mathematical Society $0002-9947 / 81 / 0000-0262 / \$ 03.25$ 
a continuous imbedding. The notation $A \approx B$, where $A$ and $B$ are norms, means that $C_{1} A \leqslant B \leqslant C_{2} B$ for some positive constants $C_{1}, C_{2}$.

1. General results. We consider a Banach space $X$ of tempered distributions in $\mathbf{R}^{n}$, i.e. $X \subset \mathcal{S}^{\prime}$.

REMARK. Sometimes we are forced to work modulo polynomials of some fixed degree, but in order not to complicate things we presently disregard this.

If $x \in \mathbf{R}^{n}$ and $h \in \mathbf{R}^{n}$ we put

$$
\langle x, h\rangle=\sum_{i=1}^{n} x_{i} h_{i} \text { and } \chi_{h}(x)=e^{i\langle x, h\rangle} .
$$

We are interested in subspaces of $X$ invariant under multiplication by $\chi_{h}$. There is a largest such space which we denote by $\pi \cdot X$, i.e. $f \in \pi \cdot X$ iff $f \in \mathcal{S}^{\prime}$ and $\chi_{h} f \in X$ for each $h \in \mathbf{R}^{n}$. On $\pi \cdot X$ we have a natural topology given by the norms $f \rightarrow\left\|\chi_{h} f\right\|$ where $h$ ranges over $\mathbf{R}^{n}$.

We further denote by $\pi_{c} X$ the space of all $f \in \mathcal{S}^{\prime}$ such that $\chi_{h} f$ is a bounded set in $X$ when $h$ belongs to a compact set in $\mathbf{R}^{n}$. We give this space the topology defined by the norms $f \rightarrow \sup _{h \in K}\left\|\chi_{h} f\right\|$ where $K$ runs over all compact subsets of $\mathbf{R}^{n}$.

Finally we want to put global restriction on $\left\|\chi_{h} f\right\|$. If $\omega$ is a given positive function on $\mathbf{R}^{n}$ we let $\pi_{\omega(h)} X$ be the space of all $f \in \pi \cdot X$ satisfying $\sup _{h \in \mathbf{R}^{n}}\left\|\chi_{h} f\right\| / \omega(h)<\infty$. With the norm $f \rightarrow \sup _{h \in \mathbf{R}^{n}}\left\|\chi_{h} f\right\| / \omega(h), \pi_{\omega(h)} X$ becomes a Banach space. With no loss of generality we may assume that $\omega$ is submultiplicative, i.e. $\omega\left(h_{1}+h_{2}\right) \leqslant \omega\left(h_{1}\right) \omega\left(h_{2}\right)$. However the only case of real interest for us is $\omega(h)=\left(1+|h|^{2}\right)^{s / 2}, s \geqslant 0$. Therefore we right away introduce the abbreviation

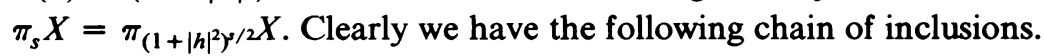

$$
X \supset \pi \cdot X \supset \pi_{c} X \supset \pi_{s} X \supset \pi_{s^{\prime}} X \quad\left(s^{\prime} \leqslant s\right) .
$$

As we will see in $\S 3$, in general we cannot expect equality here. It is also clear that, for instance, $X_{1} \subset X_{2} \Rightarrow \pi \cdot X_{1} \subset \pi \cdot X_{2}$, and similarly for $\pi_{c}$ and $\pi_{\omega(h)}$.

We now establish further properties of these spaces.

LEMMA 1. Let $G$ be a group of affine transformations on $\mathbf{R}^{n}$ which acts continuously on $X$. Then $G$ acts continuously on $\pi \cdot X$. In particular, if $X$ is translation (dilation) invariant, $\pi \cdot X$ is translation (dilation) invariant.

Proof. Let $a \in G$. Then we can write $a x=\tau+A x$ where $\tau \in \mathbf{R}^{n}$ and $A$ is a nonsingular linear transformation. Let $f \in \pi \cdot X$ and take $h \in \mathbf{R}^{n}$. Then we have the formula

$$
\chi_{h} a(f)=\exp \left(-i\left\langle A^{-1} \tau, h\right\rangle\right) a\left(\chi_{\left(A^{\prime}\right)^{-1} h} f\right) .
$$

Therefore $\chi_{h} a(f) \in X$ for each $h \in \mathbf{R}^{n}$, i.e. $a(f) \in \pi \cdot X$. The continuity is obvious.

LEMMA 2. Assume that $X$ is relatively closed in $\mathscr{D}^{\prime}$ in the sense of Gagliardo [3] (i.e. if $\left(\varphi_{\nu}\right)_{\nu \in Z}$ is a bounded sequence in $X$ which converges to $\varphi$ in $\mathscr{D}^{\prime}$ then $\left.\varphi \in X\right)$. Let $f \in \pi_{\omega(h)} X$ and $\varphi \in \mathcal{S}$ with $\int \omega(h)|\hat{\varphi}(h)| d h<\infty$. Then $\varphi f \in X$. 
Proof. For any linear combination of characters $\chi_{h}$ we have the inequality:

$$
\left\|\sum c_{i} \chi_{h_{i}} f\right\| \leqslant \sum\left|c_{i}\right| \omega\left(h_{i}\right)\|f\|_{\pi_{\omega(h)} x} .
$$

By Fourier's inversion formula,

$$
\varphi(x)=(2 \pi)^{-n} \int e^{i\langle x, h\rangle} \hat{\varphi}(h) d h .
$$

If we approximate the integral with suitable Riemann sums we get a sequence $\left(\varphi_{\nu}\right)_{\nu \in Z}$ of finite linear combinations of characters which converge to $\varphi$ in $C^{\infty}$. It follows then that $\varphi_{v} f \rightarrow \varphi f$ in $\mathscr{D}^{\prime}$. Moreover (1) shows that $\left(\varphi_{v} f\right)_{v \in Z}$ is bounded in $X$. As $X$ is relatively closed in $\mathscr{D}^{\prime}$ we may conclude that $\varphi f \in X$.

ReMark. The assumptions of Lemma 2 are, in particular, fulfilled if $X$ is a dual space. If $f \in \pi_{C} X$ and supp $\hat{\varphi}$ is compact the above proof also yields $\varphi f \in X$. (This will be needed in Theorem 3.)

2. Some function spaces. In this section we define some of the spaces which will be needed.

2.1. Besov spaces (see [1], [8]). Let $\left(\varphi_{\nu}\right)_{\nu \in Z}$ be a family of testfunctions such that:

$\varphi_{\nu} \in \mathcal{S}, \operatorname{supp} \hat{\varphi}_{\nu} \subset\left\{2^{\nu-1}<|\xi| \leqslant 2^{\nu+1}\right\}$,

$\left|\hat{\varphi}_{\nu}(\xi)\right|>C_{\varepsilon}>0$ if $2^{\nu}(2-\varepsilon)^{-1}<|\xi|<2^{\nu}(2-\varepsilon)$, for each $\varepsilon>0$,

$\left|D^{\alpha} \hat{\varphi}_{\nu}(\xi)\right| \leqslant c_{\alpha}|\xi|^{-|a|}$ for every multi-index $\alpha$.

Without loss of generality we may assume that, for a suitable $\varphi_{0}$,

$$
\varphi_{\nu}(x)=2^{\nu n} \varphi_{0}\left(2^{\nu} x\right) .
$$

In what follows $s, p, q$ will always denote numbers such that $s \in \mathbf{R}, 1<p, q<\infty$. We then define the homogeneous Besov space $\dot{B}_{p}^{s, q}$ to be the space of all distributions $f \in \mathcal{S}^{\prime}$ such that $\|f\|_{\dot{B}_{p}^{s, q}}<\infty$, where

$$
\|f\|_{\dot{B}_{p}^{s, q}}=\left(\sum_{-\infty}^{\infty}\left(2^{\nu s}\left\|\varphi_{\nu} * f\right\|_{p}\right)^{q}\right)^{1 / q} .
$$

Further, let $\Phi$ denote a function satisfying:

$\Phi \in \mathcal{S}, \operatorname{supp} \hat{\Phi} \subset\{|\xi| \leqslant 1\}$,

$|\hat{\Phi}(\xi)| \geqslant C_{\varepsilon}>0$ if $|\xi| \leqslant 1-\varepsilon$, for each $\varepsilon>0$,

$\hat{\Phi}(\xi)=1$ if $|\xi| \leqslant \frac{1}{2}$.

Moreover, we define $\Phi_{\nu}$ by $\Phi_{\nu}(x)=2^{\nu n} \Phi\left(2^{\nu} x\right)$. The inhomogeneous Besov space $B_{p}^{s, q}$ is now the space of all $f \in \mathcal{S}^{\prime}$ such that $\|f\|_{B_{p}^{s, q}}<\infty$ where

$$
\|f\|_{B_{p}^{s, q}}=\|\Phi * f\|_{p}+\left(\sum_{1}^{\infty}\left(2^{v s}\left\|\varphi_{\nu} * f\right\|_{p}\right)^{q}\right)^{1 / q} .
$$

Finally we introduce, mainly for technical reasons, the space $\mathfrak{B}_{p}^{s, q}$. Put

$$
\|f\|_{\Phi_{p}^{s, q}}=\left(\sum_{-\infty}^{\infty}\left(2^{\nu s}\left\|\Phi_{\nu} * f\right\|_{p}\right)^{q}\right)^{1 / q} .
$$

Then $\mathscr{B}_{p}^{s, q}$ is the space of all $f \in \mathcal{S}^{\prime}$ such that $\|f\|_{\mathscr{P}_{p}^{s, q}}<\infty$.

The following proposition gives some relations between these spaces. 
Proposition 1. (i) $\mathscr{B}_{p}^{s, q}=\dot{B}_{p}^{s, q}$ if $s<0$.

(ii) $\mathfrak{B}_{p}^{0, \infty}=L^{p}$ if $p>1, \mathfrak{B}_{1}^{0, \infty}=\mathfrak{N}$.

(iii) $\mathscr{H}_{p}^{s, q}=0$ if $s>0$ or $s=0$ and $q<\infty$.

(iv) $B_{p}^{s, q}=L_{p} \cap \dot{B}_{p}^{s, q}$ if $s>0$.

Proof. (i) We begin by proving $\mathscr{B}_{p}^{s, q} \subset \dot{B}_{p}^{s, q}$. We have $\varphi_{\nu}=\Phi_{\nu+1} * \varphi_{\nu}$, as is easily seen by taking Fourier transforms. Young's inequality then yields

$$
\left\|\varphi_{\nu} * f\right\|_{p} \leqslant\left\|\varphi_{\nu}\right\|_{1}\left\|\Phi_{\nu+1} * f\right\|_{p} \leqslant C\left\|\Phi_{\nu+1} * f\right\|_{p} \text {. }
$$

This immediately implies that

$$
\|f\|_{\dot{B}_{p}^{s, q}} \leqslant C\|f\|_{\mathscr{B}_{p}^{s, q}} \text {. }
$$

Conversely we now prove that $\dot{B}_{p}^{s, q} \subset \mathscr{B}_{p}^{s, q}$ if $s<0$. Assuming, in addition, that $\Sigma_{-\infty}^{\infty} \hat{\varphi}_{\nu}(\xi)=1$, we obtain as above that $\Phi_{\nu}=\Sigma_{\mu<\nu+1} \varphi_{\mu} * \Phi_{\nu}$. By application of the triangle inequality and Young's inequality we obtain

$$
\begin{aligned}
2^{\nu s}\left\|\Phi_{\nu} * f\right\|_{p} & \leqslant \sum_{\mu<\nu+1} 2^{\nu s}\left\|\Phi_{\nu}\right\|_{1}\left\|\varphi_{\mu} * f\right\|_{p} \\
& \leqslant C \sum_{\lambda>-1} 2^{\lambda s}\left(2^{(\nu-\lambda) s}\left\|\varphi_{\nu-\lambda} * f\right\|_{p}\right) .
\end{aligned}
$$

Minkowsky's inequality now implies that

$$
\|f\|_{\mathfrak{B}_{p}^{s, q}} \leqslant C\left(\sum_{\lambda>-1} 2^{\lambda s}\right)\|f\|_{B_{p}^{s, q}}
$$

where the geometrical sum converges since $s<0$.

(ii) If $f \in \mathscr{B}_{p}^{0, \infty}$ we have $\sup _{\nu}\left\|\Phi_{\nu} * f\right\|_{p}<C$. Since $\Phi_{\nu} * f \rightarrow f$ in $\mathcal{S}^{\prime}$ it follows by a classical argument involving weak compactness that $f \in L^{p}$ if $p>1$ and $f \in \mathfrak{N}$ if $p=1$.

(iii) If $s>0$ we may clearly assume that $q=\infty$. For $f \in \mathscr{B}_{p}^{s, \infty}$ it follows that $\left\|\Phi_{\nu} * f\right\|_{p} \leqslant C 2^{-\nu s}$. Thus $\Phi_{\nu} * f \rightarrow 0$ in $L^{p}$. But as in (ii), $\Phi_{\nu} * f \rightarrow f$ in $\mathcal{S}^{\prime}$ and therefore $f \equiv 0$ if $s>0$. The case $s=0, q<\infty$ is handled similarly.

(iv) See [1, p. 148].

2.2. Sobolev spaces (see [8]). Let $k \in Z_{+}$and $1<p<\infty$. The Sobolev space $W_{k}^{p}$ is then the space of all $f \in \mathcal{S}^{\prime}$ such that $D^{\alpha} f \in L^{p}$ for $|\alpha|<k$.

2.3. Spaces of Morrey type (see [6], [7]). In what follows let $\lambda>0,1<p<\infty$. If $f \in L_{\text {loc }}^{1}, x \in \mathbf{R}^{n}$ and $r>0$ we put

$$
G_{\lambda}^{p}(f, x, r)=\left(r^{-\lambda} \int_{|x-y|<r}|f(y)|^{p} d y\right)^{1 / p} .
$$

The space $M_{q}^{p, \lambda}(\cdot)$ is defined to consist of all $f \in L_{\text {loc }}^{1}$ such that for each $x \in \mathbf{R}^{n}$ the norm

$$
\|f\|_{M_{q}^{p \lambda}(\cdot), x}=\left(\sum_{-\infty}^{\infty}\left(G_{\lambda}^{p}\left(f, x, 2^{\nu}\right)\right)^{q}\right)^{1 / q}
$$

is finite. We equip $M_{q}^{p, \lambda}(\cdot)$ with the topology given by the totality of these norms as $x$ ranges over $\mathbf{R}^{n}$. 
In analogy with $\S 1$ we now introduce the space $M_{q}^{p, \lambda}(C)$. A function $f \in L_{\text {loc }}^{1}$ belongs to $M_{q}^{p, \lambda}(C)$ iff the norms

$$
\|f\|_{M_{q}^{p, \lambda}(C), K}=\left(\sum_{-\infty}^{\infty}\left(\sup _{x \in K} G_{\lambda}^{p}\left(f, x, 2^{\nu}\right)\right)^{q}\right)^{1 / q}
$$

are finite for every compact subset $K$ in $\mathbf{R}^{n}$. Using these norms we define a topology on $M_{q}^{p, \lambda}(C)$.

Let $\omega$ be a given positive function on $\mathbf{R}^{n}$. The space $M_{q}^{p, \lambda}(\omega(x))$ then consists of all $f \in L_{\text {loc }}^{1}$ satisfying

$$
\|f\|_{M_{q}^{p \lambda}(\omega(x))}=\left(\sum_{-\infty}^{\infty}\left(\sup _{x \in \mathbf{R}^{n}} G_{\lambda}^{p}\left(f, x, 2^{\nu}\right) / \omega(x)\right)^{q}\right)^{1 / q}<\infty .
$$

With this norm $M_{q}^{p, \lambda}(\omega(x))$ will be a Banach space. In particular, if $\omega(x)=$ $\left(1+|x|^{2}\right)^{s / 2}, s>0$, which is the only case of interest for us, we write $M_{q}^{p \lambda}(s)$ instead of $M_{q}^{p, \lambda}\left(\left(1+|x|^{2}\right)^{s / 2}\right)$. The usual Morrey spaces correspond to $M_{q}^{p, \lambda}(0)$ in our notation. They agree with the Stampacchia spaces $L_{q}^{p, \lambda}$ if $0<\lambda<n, q<\infty$ or $0<\lambda \leqslant n, q=\infty$. See [2] and [7]. We clearly have the following inclusions.

$$
M_{q}^{p, \lambda}(\cdot) \supset M_{q}^{p, \lambda}(C) \supset M_{q}^{p, \lambda}(s)
$$

Proposition 2. (i) $M_{q}^{p, \lambda}(\cdot)=0$ if $\lambda>n$ or $\lambda=n$ and $q<\infty$, or $\lambda=0$ and $q<\infty$.

(ii) $M_{\infty}^{p, n}(0)=L^{\infty}$.

(iii) $M_{\infty}^{p, 0}(\cdot)=M_{\infty}^{p, 0}(0)=L^{p}$.

Proof. (i) If $\lambda>n$ we may assume that $q=\infty$. Take $f \in M_{\infty}^{p, \lambda}(\cdot)$ and let $x$ be a Lebesgue point for $f$. For some $C$ we have

$$
\frac{1}{r^{n}} \int_{|x-y|<r}|f(y)|^{p} d y<C r^{\lambda-n} .
$$

Lebesgue's theorem then implies that the left side approaches $|f(x)|$ as $r \rightarrow 0$. Thus $f(x) \equiv 0$ a.e. if $\lambda>n$.

The case $\lambda=n, q<\infty$ is treated similarly. With $x$ a Lebesgue point as before, then for some $\nu_{0}$ we must have

$$
\left(\frac{1}{2^{\nu n}} \int_{|x-y|<2^{\nu}}|f(y)|^{p} d y\right)^{q / p} \geqslant \frac{1}{2}|f(x)|^{q}
$$

if $\nu \leqslant \nu_{0}$. Summing over $\nu$ we see that $f$ must vanish a.e. The case $\lambda=0, q<\infty$ is trivial.

(ii) If $f \in M_{\infty}^{p, n}(0)$ we may choose $C$ in (2) independent of $x$. Letting $\nu \rightarrow-\infty$ we see that $f \in L^{\infty}$, again by Lebesgue's theorem.

(iii) Trivial.

3. Determination of $\pi \cdot \dot{B}_{p}^{s, q}$. We now turn to the main topic of this paper. Since the three cases $s>0, s=0$ and $s<0$ behave quite differently, we divide this section into three parts.

3.1. The case $s>0$. 
Theorem 1. If $s>0$ and $1 \leqslant p, q \leqslant \infty$ then $\pi \cdot \dot{B}_{p}^{s, q}=B_{p}^{s, q}$. Moreover we have for each $h \in \mathbf{R}^{n}$ and $f$ in this space

$$
\|f\|_{\dot{B}_{p}^{s, q}}+\left\|\chi_{h} f\right\|_{\dot{B}_{p}^{s, q}} \approx|h|^{s}\|f\|_{p}+\|f\|_{\dot{B}_{p}^{s, q} .}
$$

The main component in the proof will be the observation that multiplication by $\chi_{h}$ corresponds to translation on the Fourierside, which is expressed in the following lemma.

Lemma 3. Assume that $\psi \in L^{1}$ and supp $\hat{\psi}$ is compact. For some $\nu_{0}$ we then have the inequality

$$
\|\psi * f\|_{p} \leqslant\|\psi\|_{1}\left\|\varphi_{\nu} * \chi_{h} f\right\|_{p}
$$

if $\nu \geqslant \nu_{0},|h| \approx 2^{\nu}$ and the right-hand side is finite, $1<p<\infty$.

Proof of Lemma 3. Choose $\left(\varphi_{\nu}\right)_{\nu \in Z}$, as in the definition of $\dot{B}_{p}^{s, q}$, satisfying, in addition, $\hat{\varphi}_{\nu}(\xi)=1$ if $\xi \in I_{\nu}=\left\{\xi:|| \xi\left|-2^{\nu}\right|<C_{0} 2^{\nu}\right\}$. As supp $\hat{\psi}$ is compact, there exists a $\nu_{0}$ such that $\operatorname{supp} \hat{\psi} \subset\left\{|\xi|<C_{0} 2^{\nu_{0}}\right\}$. Thus if $\nu>\nu_{0},|h| \approx 2^{\nu}$, we have $\operatorname{supp}\left(\chi_{h} \psi\right)^{\hat{n}}=h+\operatorname{supp} \hat{\psi} \subset I_{\nu}$. Our choice of $\left(\varphi_{\nu}\right)_{\nu \in Z}$ then implies that $\chi_{h} \psi * \varphi_{\nu}=$ $\chi_{h} \psi$. Rewriting $\chi_{h}(\psi * f)$ as $\varphi_{\nu} * \chi_{h} \psi * \chi_{h} f$ and applying Young's inequality, we get

$$
\|\psi * f\|_{p}=\left\|x_{h}(\psi * f)\right\|_{p} \leqslant\|\psi\|_{1}\left\|\varphi_{\nu} * \chi_{h} f\right\|_{p}
$$

Proof of Theorem 1. We begin by proving (3) for a fixed $h$. With $\psi=\Phi$, as in the definition of $B_{p}^{s, q}$, and $h_{0}$ such that $\left|h_{0}\right| \approx 1$ we obtain from Lemma 3,

$$
\|\Phi * f\|_{p} \leqslant C\left\|\chi_{h_{0}} f\right\|_{\dot{B}_{p}^{s, q}}
$$

This implies that

$$
\|f\|_{B_{p}^{s, q}} \leqslant C\left\|\chi_{h_{0}} f\right\|_{\dot{B}_{p}^{s, q}}+\|f\|_{\dot{B}_{p}^{s, q} .}
$$

In order to obtain the converse inequality we first use the imbedding $B_{p}^{s, q} \subset \dot{B}_{p}^{s, q}$, $s>0$ (Proposition 1(iv)) which gives

$$
\begin{aligned}
\|f\|_{\dot{B}_{p}^{s, q}} & \leqslant C\|f\|_{B_{p}^{s, q}} \\
\left\|\chi_{h_{0}} f\right\|_{\dot{B}_{p}^{s, q}} & \leqslant C\left\|\chi_{h_{0}} f\right\|_{B_{p}^{s, q} \cdot}
\end{aligned}
$$

But $\chi_{h}$ acts continuously on $B_{p}^{s, q}$. Indeed, in view of the fact that $B_{p}^{s, q}=$ $\left(L^{p}, W_{k}^{p}\right)_{\theta, q}, s=\theta k, 0<\theta<1$ (see [8, p. 64]), it suffices to show that $\chi_{h}$ acts continuously on $W_{k}^{p}$, which is obvious. We thus have

$$
\left\|\chi_{h_{0}} f\right\|_{\dot{B}_{p}^{s, q}} \leqslant C\|f\|_{B_{p}^{s, q} \text {. }}
$$

By combining (4)-(6) we get the desired inequalities:

$$
\|f\|_{B_{p}^{s, q}} \approx\left\|\chi_{h_{0}} f\right\|_{B_{p}^{s, q}}+\|f\|_{\dot{B}_{p}^{s, q}} \quad \text { with }\left|h_{0}\right| \approx 1 \text {. }
$$

An argument with dilations will now establish (3). Indeed take $0 \neq h \in \mathbf{R}^{n}$. As rotations act continuously on $\dot{B}_{p}^{s, q}$ (see Lemma 1 ) we may assume that $h$ and $h_{0}$ are collinear, i.e. $h=\lambda h_{0}$ for some $\lambda>0$.

Let $\tau_{\delta}$ denote the dilation operator defined by

$$
\left(\tau_{\delta} f\right)(x)=f(\delta x), \quad \delta>0 .
$$


It is well known that $\tau_{\delta}$ acts continuously on $\dot{B}_{p}^{s, q}$ and we have

$$
\left\|\tau_{\delta} f\right\|_{\dot{B}_{p}^{s, q}} \approx \delta^{s-n / p}\|f\|_{\dot{B}_{p}^{s, q}}
$$

We further notice the formula

$$
\chi_{h} f=\tau_{\lambda}\left(\chi_{h_{0}} \tau_{1 / \lambda} f\right) .
$$

If we apply (7) with $\tau_{1 / \lambda} f$ and multiply by $\lambda^{s-n / p}$ we get, in view of (8) and (9),

$$
\left\|\chi_{h} f\right\|_{\dot{B}_{p}^{s, q}}+\|f\|_{\dot{B}_{p}^{s, q}} \approx \lambda^{s-n / p}\left\|\tau_{1 / \lambda} f\right\|_{B_{p}^{s, q}}
$$

Finally, the right side is simplified by once again invoking Proposition 1(iv) thereby obtaining

$$
\begin{aligned}
\lambda^{s-n / p}\left\|\tau_{1 / \lambda} f\right\|_{B_{p}^{s, q}} & \approx \lambda^{s-n / p}\left(\left\|\tau_{1 / \lambda} f\right\|_{p}+\left\|\tau_{1 / \lambda} f\right\|_{\dot{B}_{p}^{s, q}}\right) \\
& \approx \lambda^{s}\|f\|_{p}+\|f\|_{\dot{B}_{p}^{s, q} .}
\end{aligned}
$$

As $\lambda^{s} \approx|h|^{s}$ we have now proved (3), and thus $\pi \cdot \dot{B}_{p}^{s, q}=B_{p}^{s, q}$.

Corollary 1. Let $s>0,1 \leqslant p, q \leqslant \infty$. Then $\pi_{s^{\prime}} \dot{B}_{p}^{s, q}=B_{p}^{s, q}$ if $s^{\prime}>s, \pi_{s^{\prime}} \dot{B}_{p}^{s, q}=0$ if $s^{\prime}<s$.

2.2. The case $s=0$.

THEOREM 2. (i) If $1 \leqslant p, q \leqslant \infty$ then $\pi \cdot \dot{B}_{p}^{0, q} \subset B_{p}^{0, q}$.

(ii) If $q=\infty$ and $1 \leqslant p \leqslant \infty$ or $q \geqslant \max (2, p)$ and $1<p<\infty$, then

$$
\pi_{0} \dot{B}_{p}^{0, q}=L^{p} \quad \text { if } p>1, \pi_{0} \dot{B}_{1}^{0, \infty}=\Re \text {. }
$$

Proof. (i) As in the proof of Theorem 1 we obtain

$$
\|f\|_{B_{p}^{0, q}} \leqslant C\left\|\chi_{h} f\right\|_{\dot{B}_{p}^{0, q}}+\|f\|_{\dot{B}_{p}^{0 . q}} \quad \text { if }|h| \approx 1 .
$$

Thus $\pi \cdot \dot{B}_{p}^{0, q} \subset B_{p}^{0, q}$.

(ii) If $p$ and $q$ are as in the hypothesis, we have the imbedding $L^{p} \subset \dot{B}_{p}^{0, q}$ (see [8, p. 80]). It follows that $L^{p} \subset \pi_{0} \dot{B}_{p}^{0, q}$. We now show that, conversely, $\pi_{0} \dot{B}_{p}^{0, q} \subset L^{p}$ holds. Take $f \in \pi_{0} \dot{B}_{p}^{0, q}$. Then for each $\nu, \Phi_{\nu} * f \in \pi_{0} \dot{B}_{p}^{0, q}$. Lemma 3 now gives, if $|h| \approx 2^{\nu}$ and $\psi=\Phi_{\nu+1}$,

$$
\begin{aligned}
\left\|\Phi_{\nu} * f\right\|_{p} & \leqslant C\left\|\chi_{h}\left(\Phi_{\nu} * f\right)\right\|_{\dot{B}_{p}^{0, q}} \\
& \leqslant C\left\|\Phi_{\nu} * f\right\|_{\pi_{0} \dot{B}_{p}^{0, q}} \\
& \leqslant C\|f\|_{\pi_{0} \dot{B}_{p}^{0 . q}}
\end{aligned}
$$

so by Proposition 1(ii), $f \in L^{p}$ if $p>1 . f \in \mathfrak{T}$ if $p=1$.

2.3. The case $s<0$. We first observe that Proposition 1(ii) allows us to replace $\dot{B}_{p}^{s, q}$ with $\mathfrak{B}_{p}^{s, q}$ as $s<0$. This will be done in the proofs given below. It will be convenient to introduce the following terminology.

Definition. We say that $s, p, q$, where $s<0,1<p, q<\infty$, are good indices iff $-n / p^{\prime}<s<0$ if $q=\infty$, or $-n / p^{\prime}<s<0$ if $q<\infty$. ( $p^{\prime}$ denotes the conjugate index to $p$.) Otherwise we say that $s, p, q$ are bad indices.

Using this notation we now state our main result. 
THEOREM 3. If $s, p$, $q$ are bad indices then

(i) $\pi \cdot \dot{B}_{p}^{s, q} \cap L^{1}=0$,

(ii) $\pi_{c} \dot{B}_{p}^{s, q}=0$.

Proof. We reduce to the case $p=\infty$. This may be accomplished by using Besov's imbedding theorem (see [8, p. 63]) implying that $\dot{B}_{p}^{s, q} \subset \dot{B}_{\infty}^{s-n / p, q}$.

(i) Take $f \in \pi \cdot \dot{B}_{\infty}^{s, q} \cap L^{1}$. We then obtain, using Parseval's formula,

$$
\begin{aligned}
2^{\nu s}\left\|\Phi_{\nu} * f\right\|_{\infty} & \geqslant 2^{\nu s}\left|\left(\Phi_{\nu} * f\right)(0)\right|=2^{\nu s}\left|\int \Phi_{\nu}(x) f(-x) d x\right| \\
& =C 2^{\nu s}\left|\int \hat{\Phi}\left(\eta / 2^{\nu}\right) \hat{f}(-\eta) d \eta\right| .
\end{aligned}
$$

We may assume that $\hat{\Phi}(\eta) \geqslant 0$. If we apply the above inequality to $\chi_{n}(f * \bar{f})$ and then use Young's inequality we find that

$$
2^{\nu s} \int_{|\eta-h|<2^{\nu-1}}|\hat{f}(\eta)|^{2} d \eta \leqslant C 2^{\nu s}\left\|\Phi_{\nu} * \chi_{h} f\right\|_{\infty}\|f\|_{1}
$$

Therefore it follows that $\hat{f} \in M_{2 q}^{2,-s}(\cdot)$. After invoking Proposition 2(ii), we arrive at the result $\hat{f} \equiv 0$ if $s, \infty, q$ are bad indices.

(ii). Take $f \in \pi_{c} \dot{B}_{\infty}^{s, q}$. Our aim is to modify $f$ so that we may use (i). Assume first that $q>1$. Take $\psi$ with $\hat{\psi} \in C_{0}^{\infty}$. Then $f * \psi \in \pi_{c} \dot{B}_{\infty}^{s, q}$. As $\operatorname{supp}(\psi * f)^{\wedge}$ is compact, it is easily seen that $f * \psi \in L^{\infty}$. Indeed we clearly have for some finite $N$,

$$
f * \psi=\sum_{-\infty}^{N} \varphi_{\nu} * f * \psi
$$

As $s<0$, an application of Minkowsky's inequality yields

$$
\|f * \psi\|_{\infty} \leqslant\|f * \psi\|_{\dot{B}_{\infty}^{0.1}} \leqslant 2^{-N s}\|f * \psi\|_{B_{\infty}^{s . q}}
$$

Take $\varphi \in L^{1} \cap \mathcal{S}$ with supp $\hat{\varphi}$ compact. Hölder's inequality now implies that $\varphi(f * \psi) \in L^{1}$. As $\dot{B}_{\infty}^{s, q}$ is a dual space for $q>1$ (see [8, p. 74]), we find, by using Lemma 2, that $\varphi(f * \psi) \in \dot{B}_{\infty}^{s, q}$. Thus $\varphi(f * \psi) \in \pi \cdot \dot{B}_{\infty}^{s, q} \cap L^{1}$ if we apply the above argument to $\chi_{h} \varphi(f * \psi)$. (i) then shows that $\varphi(f * \psi) \equiv 0$ for all $\varphi$ and $\psi$. Thus $f$ must vanish.

The case $q=1$ follows trivially from what we have proved for $q>1$. Just notice that $\dot{B}_{p}^{s, 1} \subset \dot{B}_{p}^{s, q}$ for any $q \geqslant 1$.

The main idea in this proof was the pointwise behaviour of $f$. The HausdorffYoung theorem allows us to sharpen Theorem 2 if $1<p<2$. Let $\mathscr{F}$ denote the Fourier transform.

Theorem 4. Let $s<0,1 \leqslant p \leqslant 2,1 \leqslant q \leqslant \infty$. Then $\mathscr{F}: \pi \cdot \dot{B}_{p}^{s, q} \rightarrow M_{q}^{p^{\prime},-s p^{\prime}}(\cdot)$. In particular, $\pi \cdot \dot{B}_{p}^{s, q}=0$ if $s, p, q$ are bad indices.

Proof. An application of the Hausdorff-Young theorem yields for each $h \in \mathbf{R}^{n}$ and $\nu$,

$$
\begin{aligned}
2^{\nu s}\left\|\Phi_{\nu} * \chi_{h} f\right\|_{p} & \geqslant C 2^{\nu s}\left\|\hat{\Phi}\left(\cdot / 2^{\nu}\right) \hat{f}(\cdot-h)\right\|_{p^{\prime}} \\
& \geqslant C\left(2^{\nu s p^{\prime}} \int_{|\eta-h|<2^{\nu-1}}|\hat{f}(\eta)|^{p^{\prime}} d \eta\right)^{1 / p^{\prime}} \\
& =C G_{-s p^{\prime}}^{p^{\prime}}\left(\hat{f}, h, 2^{\nu-1}\right) .
\end{aligned}
$$


Raising this to the $q$ th power and summing over $\nu$ we obtain

$$
\|\hat{f}\|_{M_{q}^{p^{\prime}-\boldsymbol{s}^{\prime}(\cdot), h}} \leqslant C\left\|\chi_{h} f\right\|_{\dot{B}_{p}^{s, q} .}
$$

This proves the first part of the theorem.

That $\pi \cdot \dot{B}_{p}^{s, q}=0$, for $s, p, q$ bad indices, is now a consequence of Proposition 2(i).

Conversely, reversing all inequality signs in the above proof, we obtain the following theorem and corollary.

TheOREM 5. If $s<0,2 \leqslant p \leqslant \infty, 1 \leqslant q \leqslant \infty$. Then $\mathscr{F}: M_{q}^{p^{\prime},-s p^{\prime}}(\cdot) \rightarrow \pi \cdot \dot{B}_{p}^{s, q}$.

Corollary 2. If $s<0,1 \leqslant q \leqslant \infty$ then

$$
\pi \cdot \dot{B}_{2}^{s, q}=\mathscr{F} M_{q}^{2,-2 s}(\cdot) .
$$

Remarks. (i) Theorems 4 and 5 and Corollary 2 have obvious extensions to the spaces $\pi_{\omega(h)} \dot{B}_{p}^{s, q}$ and $\pi_{c} \dot{B}_{p}^{s, q}$. The conclusions will then involve the spaces $M_{q}^{p, \lambda}(\omega(x))$ and $M_{q}^{p, \lambda}(C)$.

(ii) Corollary 2 shows that the spaces $\pi \cdot X, \pi_{c} X$ and $\pi_{\omega(h)} X$ do not coincide in general. For instance it is easily seen that $M_{q}^{2,-2 s}(\cdot)$ and $M_{q}^{2,-2 s}(C)$ behave topologically differently. Further, $f \in M_{\infty}^{2, n}(\omega(x))$ implies that $|f(x)|<C \omega(x)$. As $M_{\infty}^{2, n}(0)=L^{\infty}$ we thus see that $\pi_{\omega(h)} \dot{B}_{2}^{-n / 2, \infty} \neq \pi_{0} \dot{B}_{2}^{-n / 2, \infty}$ if, e.g., $\omega(h) \rightarrow 0$ as $h \rightarrow \infty$.

As is seen by Theorem $5, \pi \cdot \dot{B}_{p}^{s, q}$ is a "large" space if $2<p \leqslant \infty$ and $s, p, q$ are good indices. This is true without any restrictions on $p$, as is seen from the following proposition.

Proposition 3. $\delta \subset \pi_{0} \dot{B}_{p}^{s, q}$ if $s, p, q$ are good indices.

We omit the proof since this simply amounts to a use of Young's inequality.

REMARK. Some of our results are already found in [4]. More precisely, Johnson considers $\pi_{s} \dot{B}_{p}^{s, q}$ if $s>0$ and $\pi_{0} \dot{B}_{p}^{s, \infty}$ if $s \leqslant 0$. He establishes a weaker version of Theorem 1. He also proves Theorem 2(ii), if $q=\infty$, Theorems 4, 5 and Corollary 2 for $\pi_{0} \dot{B}_{p}^{s, \infty}$.

\section{REFERENCES}

1. J. Bergh and J. Löfström, Interpolation spaces. An introduction, Springer, Berlin and New York, 1976.

2. S. Campanato, Proprietà di una famiglia di spazi funzionali, Ann. Scuola Norm. Sup. Pisa 18 (1964), 137-160.

3. E. Gagliardo, $A$ unified structure in various families of function spaces. Compactness and closure theorems, Proc. Internat. Sympos. Linear Spaces (Jerusalem, 1960), Jerusalem Academic Press, Jerusalem; Pergamon, Oxford, 1961.

4. R. Johnson, Maximal subspaces of Besov spaces invariant under multiplication by characters, Trans. Amer. Math. Soc. 249 (1979), 387-407.

5. __ Duality methods for the study of maximal subspaces of Besov spaces invariant under multiplication by characters, Univ. of Maryland Tech. Report 79, 75.

6. C. B. Morrey, Functions of several variables and absolute continuity, Duke Math. J. 6 (1940), 187-215.

7. J. Peetre, On the theory of $L_{p, \lambda}$ spaces, J. Funct. Anal. 4 (1969), 71-87.

8. __ New thoughts on Besov spaces, Duke Univ. Math. Ser. Durham, N. C., 1976.

DePartment of MAThematics, UnIVERStTy OF LUND, S-22007 Lund, SWEDEN 